\title{
Intensity-Modulated Radiotherapy-Based Reirradiation for Head and Neck Cancer: A Multi-institutional Study by Korean Radiation Oncology Group (KROG 1707)
}

\author{
Jeongshim Lee, MD \\ Tae Hyung Kim, MD2 \\ Yeon-Sil Kim, MD, PhD ${ }^{3}$ \\ Myungsoo Kim, MD, PhD ${ }^{4}$ \\ Jae Won Park, MD, PhD \\ Sung Hyun Kim, $\mathrm{MD}^{6}$ \\ Hyun Ju Kim, MD \\ Chang Geol Lee, MD, PhD²
}

${ }^{1}$ Department of Radiation Oncology, Inha University Hospital, Inha University College of Medicine, Incheon, ${ }^{2}$ Department of Radiation Oncology, Yonsei University College of Medicine, Seoul, ${ }^{3}$ Department of Radiation Oncology, St. Mary's Hospital, College of Medicine, The Catholic University of Korea, Seoul, ${ }^{4}$ Department of Radiation Oncology, Incheon St. Mary's Hospital, College of Medicine, The Catholic University of Korea, Seoul, ${ }^{5}$ Department of Radiation Oncology, Yeungnam University Medical Center, Yeungnam University College of Medicine, Daegu, ${ }^{6}$ Department of Radiation Oncology, Wonju Severance Christian Hospital, Yonsei University Wonju College of Medicine, Wonju, ${ }^{7}$ Department of Radiation Oncology, Gachon University Gil Medical Center, Incheon, Korea

Correspondence: Chang Geol Lee, MD, PhD Department of Radiation Oncology,

Yonsei University College of Medicine,

50 Yonsei-ro, Seodaemun-gu, Seoul 03722, Korea

Tel: 82-2-2228-8114

Fax: 82-2-312-9033

E-mail: cglee1023@yuhs.ac

Received April 14, 2020

Accepted July 6, 2020

Published Online July 7, 2020

\begin{abstract}
Purpose
The benefits of reirradiation for head and neck cancer (HNC) have not been determined. This study evaluated the efficacy of reirradiation using intensity-modulated radiotherapy (IMRT) for recurrent or second primary HNC (RSPHNC) and identified subgroups for whom reirradiation for RSPHNC is beneficial.
\end{abstract}

\section{Materials and Methods}

A total of 118 patients from seven Korean institutions with RSPHNC who underwent IMRTbased reirradiation between 2006 and 2015 were evaluated through retrospective review of medical records. We assessed overall survival (OS) and local control (LC) within the radiotherapy (RT) field following IMRT-based reirradiation. Additionally, the OS curve according to the recursive partitioning analysis (RPA) suggested by the Multi-Institution Reirradiation (MIRI) Collaborative was determined.

\section{Results}

At a median follow-up period of 18.5 months, OS at 2 years was $43.1 \%$. In multivariate analysis, primary subsite, recurrent tumor size, interval between RT courses, and salvage surgery were associated with OS. With regard to the MIRI RPA model, the class I subgroup had a significantly higher OS than class II or III subgroups. LC at 2 years was $53.5 \%$. Multivariate analyses revealed that both intervals between RT courses and salvage surgery were prognostic factors affecting LC. Grade 3 or more toxicity and grade 5 toxicity rates were $8.5 \%$ and $0.8 \%$, respectively.

\section{Conclusion}

IMRT-based reirradiation was an effective therapeutic option for patients with RSPHNC, especially those with resectable tumors and a long interval between RT courses. Further, our patients' population validated the MIRI RPA classification by showing the difference of OS according to MIRI RPA class.

\section{Key words}

Head and neck neoplasm, Recurrence, Reirradiation, Intensity modulated radiotherapy

\section{Introduction}

Despite aggressive multidisciplinary management including radiotherapy (RT), up to half of patients with locally advanced head and neck cancer (HNC) experience locoregional recurrence [1-3]. Although surgery was the best sal- vage therapy option for such patients, only a few patients could be candidates for surgical resection due to unresectability or inoperability associated with poor performance functions and their comorbidities $[4,5]$. Thus, for these patients, reirradiation has been considered as a salvage therapy to improve outcomes, although the concern of severe toxicity 
following reirradiation has remained [6].

With the widespread adoption of conformal RT techniques such as intensity-modulated RT (IMRT) and volume-modulated arc therapy, the therapeutic ratio of reirradiation may have increased owing to the advanced technique using multiple small beamlets, which have an accurate target system for irregularly shaped tumors while simultaneously avoiding normal tissue $[7,8]$. Therefore, reirradiation in the modern advanced RT era has led to the expectation that the efficacy after reirradiation would be improved [9], and it has contributed to the frequent application of reirradiation using IMRT in clinical practice [10-12].

However, the efficacy of reirradiation in the IMRT era still remains unclear. In particular, difficulties associated with the selection of patients who would benefit from reirradiation and the potential for lethal toxicity following reirradiation are barriers to performing reirradiation. Therefore, we conducted a multi-institutional study to describe the efficacy of IMRTbased reirradiation for recurrent or second primary HNC (RSPHNC) and to identify prognostic factors for which the benefit of reirradiation appears favorable. We also sought to externally validate the recursive partitioning analysis (RPA) published by the Multi-Institution Reirradiation (MIRI) Collaborative [13].

\section{Materials and Methods}

\section{Study design}

We performed a multicenter, retrospective cohort study to assess the efficacy of IMRT-based reirradiation for HNC. The eligibility criteria were as follows: (1) adult patients ( $\geq 18$ years) who had locoregional RSPHNC without distant metastasis (M0) based on histologic and/or radiographic evidence of progression of the disease treated with IMRTbased reirradiation from January 1, 2006, to December 31, 2015; (2) availability of a medical record related to the initial radiation dose; and (3) patients who previously received $\geq 40$ Gy RT at the reirradiation site. Information on the patients' clinicodemographic characteristics, tumor characteristics, and administered treatments was collected from their medical records. All institutions decided on re-irradiation for patients with RSPHNC through each institution's multidisciplinary discussion during a head and neck tumor conference, in which an otolaryngologist, radiation oncologist, medical oncologist, and radiologist participated. Finally, 118 patients from seven institutions with recurrent $(n=109)$ or second primary $(n=9)$ HNC who underwent IMRT-based reirradiation were analyzed.

\section{Treatment outcomes and prognostic variables}

The primary endpoint was 2-year overall survival (OS) rate, and the secondary endpoint was local control (LC) rate.
OS period was calculated from the time of reirradiation for recurrence to the date of death from any cause, and freedom from local progression was defined as an absence of disease on histologic and radiographic evaluation from the reirradiation. The prognostic factors associated with OS and LC were as follows: age, sex, Karnofsky performance status, initial subsite, initial histology, surgery at recurrence, tumor size at recurrence, interval between RT courses, and organ dysfunction at reirradiation. In addition, we divided patients into prognostic subgroups using the RPA according to the interval between RT courses, resectability, and organ dysfunction defined by the MIRI Collaborative [13] and assessed OS by class. Additionally, the late toxicity following reirradiation was assessed by reviewing medical charts.

\section{Statistical analysis}

Actuarial estimates for OS and LC were calculated using the Kaplan-Meier methods. Log-rank test was used to compare risk factors affecting survival and LC estimates in univariate analysis. A Cox regression model was used to identify potential prognostic factors for OS and LC in univariate and multivariate analyses. All analyses were performed using IBM SPSS ver. 20.0 (IBM Corp., Armonk, NY).

\section{Ethical statement}

This study was approved by the institutional review board of each participating institution. The requirement for informed consent was waived. We carried out this research according to the principles expressed in the declaration of Helsinki.

\section{Results}

\section{Patient and treatment characteristics}

The median age when performing IMRT-based reirradiation was 59 years (range, 20 to 90 years). Of the total patients, 95 patients $(80.5 \%)$ had squamous cell carcinoma and 82 patients $(69.5 \%)$ showed stage III/IV at initial presentation. The approach to the RSPHNC consisted of surgery for $40.7 \%$ $(\mathrm{n}=48 ; \mathrm{R} 0$ resection in 29 patients and R1 resection in 19 patients) and chemotherapy for $72.9 \%(\mathrm{n}=86)$ of the patients. Fractionation of IMRT-based reirradiation was once daily for all patients. The treatment volume included focal field in $84.7 \%$, which was defined by the gross tumor or surgical bed plus a margin of $0.5-1.0 \mathrm{~cm}$, and radical field in $15.3 \%$, encompassing the gross tumor or surgical bed with an elective high-risk area plus a margin of 0.5-1.0 cm. The median interval period between RT courses was 29.4 months (range, 2.6 to 293.4 months). The median initial RT total dose, IMRT-based reirradiation total dose, and cumulative RT dose were $66 \mathrm{~Gy}$ (range, 40.0 to $78.6 \mathrm{~Gy}$ ), 59.4 Gy (range, 36.0 to $75.0 \mathrm{~Gy}$ ), and 124.9 Gy (range, 90.0 to $146.3 \mathrm{~Gy}$ ), respectively. With regard 
Table 1. Baseline characteristics

\section{Variable}

Total $(\mathrm{n}=118)$

\section{Initial presentation}

Age, median (range, yr)

Sex

Male $92(78.0)$

Female $26(22.0)$

ECOG PS

$0-1$

$110(93.2)$

2-3

8 (6.8)

Primary subsite

Nasopharynx $30(25.4)$

Oropharynx $15(12.7)$

Oral cavity 9 (7.6)

Paranasal sinus/Nasal cavity $23(19.5)$

Hypopharynx/Larynx 30 (25.4)

Other (SG, UP)

$11(9.3)$

Histology

SCC

$95(80.5)$

Non-SCC

$23(19.5)$

Stage

I/II $31(26.3)$

III $16(13.6)$

IV $66(55.9)$

Unevaluable

$5(4.2)$

Initial treatment

Treatment modality

Definitive RT / CRT

$52(44.1)$

Surgery \pm PORT / CRT

$48(40.7)$

Induction $\mathrm{CT} x+$ Definitive RT / CRT

$13(11.0)$

Induction CTx+Surgery \pm PORT / CRT

$3(2.5)$

Preoperative RT / CRT+Surgery

$2(1.6)$

Initial RT total dose, median (range, Gy) 66.0 (40.0-78.6)

Initial RT fractional dose,

$2.0(1.2-2.5)$

median (range, Gy)

Second presentation

Age, median (range, yr)

$<60$

$59(20-90)$

$63(53.4)$

$\geq 60$

$55(46.6)$

ECOG PS

$0-1 \quad 105.0(89.0)$

2-3 $13.0(11.0)$

Presentation type

Recurrent $109(92.4)$

SP $9(7.6)$

rStage

$\begin{array}{ll}\text { rT0Nany } & 25(21.2) \\ \text { rT1Nany } & 13(11.0) \\ \text { rT2Nany } & 11(9.3) \\ \text { rT3Nany } & 19(16.1) \\ \text { rT4Nany } & 50(42.4)\end{array}$

(Continued)

Table 1. Continued

Variable

Failure type

Local failure

Regional failure

Locoregional failure

Recurrent or SP tumor size $(\mathrm{cm})$

Median, range

$<3$

$\geq 3$

No. of recurrent or SP tumor

$1 \quad 72(61.0)$

$\geq 2$

$46(39.0)$

Pre-existing organ dysfunction

$\begin{array}{lc}\text { No } & 110(93.2) \\ \text { Yes }^{\text {a) }} & 8(6.8)\end{array}$

RPA class ${ }^{\text {b) }}$

Class I

Class II

$25(21.2)$

Class III

$89(75.4)$

4 (3.4)

Second treatment

Salvage surgery

$\begin{array}{ll}\text { No } & 70(59.3) \\ \text { Yes }^{\text {c) }} & 48(40.7)\end{array}$

No $32(27.1)$

Yes $\quad 86$ (72.9)

Reirradiation total dose (Gy)

Median (range) $\quad 59.4$ (36.0-75.0)

$<60 \quad 60(50.8)$

$\geq 60 \quad 58(49.2)$

Reirradiation fractional dose, $\quad 2.1$ (1.8-4.0)

median (range, Gy)

Treatment volume of reirradiation ${ }^{\mathrm{d})}$

Focal field

$100(84.7)$

Radical field

18 (15.3)

Cumulative RT dose,

median (range, Gy)

(Continued to the next page)

to RPA classes, class I, II, and III accounted for $21.2 \%, 75.4 \%$, and $3.4 \%$, respectively. The baseline characteristics of 118 patients from seven institutions are summarized in Table 1. Furthermore, the baseline characteristics according to each institution are shown in S1 Table.

\section{Outcomes and prognostic factors}

The median duration of follow-up after IMRT-based reirradiation was 18.5 months (range, 1.4 to 98.0 months). Twentynine patients $(24.6 \%)$ remained alive at the time of the last observation. The median OS duration and 2-year OS rate were 20.1 months (range, 16.1 to 24.1 months) and 43.1\%, 
Table 1. Continued

\begin{tabular}{lc} 
Variable & Total $(\mathbf{n}=118)$ \\
\hline Interval between RT courses (mo) & \\
Median (range) & $29.4(2.6-293.4)$ \\
$<24$ & $52(44.1)$ \\
$\geq 24$ & $66(55.9)$ \\
\hline
\end{tabular}

ECOG PS, Eastern Cooperative Oncology Group performance status; SG, salivary gland; UP, unknown primary; SCC, squamous cell carcinoma; RT, radiotherapy; CRT, chemoradiotherapy; PORT, postoperative radiotherapy; $\mathrm{CTx}$, chemotherapy; $\mathrm{SP}$, second primary; rStage, recurrent tumor and nodal stage; RPA, recursive partitioning analysis. ${ }^{a}$ Tracheostomy $(n=6,5.2 \%)$ or feeding tube dependence $(n=2,1.6 \%)$ prior to re-irradiation, ${ }^{b}$ Prognostic groups associated with overall survival according to RPA defined by the Multi-Institution Reirradiation (MIRI) Collaborative, ${ }^{\mathrm{c}}$ Salvage surgery was performed in R0 resection for 29 patients and R1 resection for 19 patients, ${ }^{\text {d) }}$ Focal field included the gross tumor or surgical bed plus margin of $0.5-1.0 \mathrm{~cm}$, whereas radical field encompassed the gross tumor or surgical bed with elective high risk area plus margin of $0.5-1.0 \mathrm{~cm}$.

respectively (Fig. 1). The Kaplan-Meier curve of factors related to OS after reirradiation identified primary subsites $(\mathrm{p}<0.001)$ (Fig. 2A), RSPHNC tumor size $(\mathrm{p}<0.001)$ (Fig. $2 B)$, number of RSPHNC ( $\mathrm{p}=0.041)$, interval between RT courses ( $\mathrm{p}=0.007$ ) (Fig. 2C), and performance of salvage surgery for RSPHNC ( $\mathrm{p}=0.002)$ (Fig. 2D) as significant factors. In a multivariate stepwise Cox regression model analysis, primary subsites (non-hypopharynx/larynx/oral cavity vs. hypopharynx/larynx/oral cavity; hazard ratio [HR], 0.457; $\mathrm{p}=0.001)$, tumor size of RSPHNC ( $\geq 3 \mathrm{~cm}$ vs. $<3 \mathrm{~cm}$; HR, 2.119; $\mathrm{p}=0.001$ ), interval between RT courses ( $\geq 24$ months vs. $<24$ months; HR, 0.460; $\mathrm{p}<0.001$ ), and salvage surgery (yes vs. no; HR, 0.586; $\mathrm{p}=0.023$ ) were confirmed as independent prognostic factors affecting OS. All results from the univariate and multivariate Cox regression analyses related to OS are shown in Table 2.

Local progression within the reirradiation field was developed in 50 patients (42.4\%). The median time to local failure and 2-year LC rate were 28.9 months (range, 8.23 to 49.57 months) and $53.5 \%$, respectively (Fig. 1). Both univariate and multivariate Cox regression models revealed that the interval between RT courses ( $\mathrm{p}=0.078$ and $\mathrm{p}=0.011$, respectively) and performance of salvage surgery for RSPHNC ( $\mathrm{p}=0.026$ and $\mathrm{p}=0.042$, respectively) were significant prognostic factors associated with LC (Table 2). Fig. 3A and Fig. 3B show the Kaplan-Meier LC curve according to the interval between RT courses $(\mathrm{p}=0.074)$ and performance of salvage surgery $(\mathrm{p}=0.023)$, respectively.

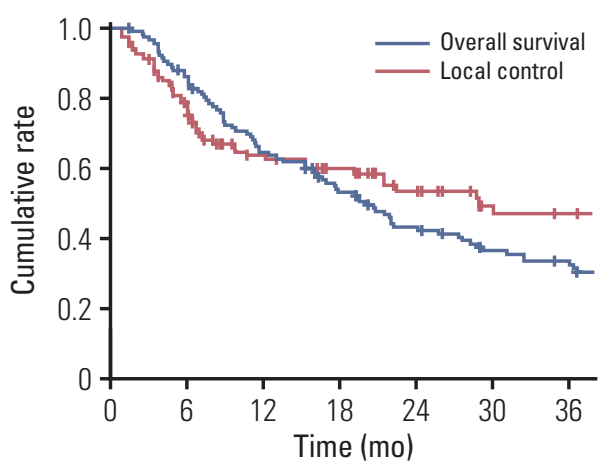

Fig. 1. Kaplan-Meier curve of overall survival and local control rates.

\section{Survival validation according to MIRI RPA}

We classified the patients into three prognostic classes according to the time interval between RT courses ( $<2$ years vs. $\geq 2$ years), resectability (resected vs. unresected), and organ dysfunction (yes vs. no), as follows: those with interval $>2$ years between RT courses with resected tumors (class I, $n=25$ ), those with interval $>2$ years between RT courses with unresected tumors or with interval $\leq 2$ years between RT courses without organ dysfunction (class II, $n=89$ ), and those with interval $\leq 2$ years between RT courses with organ dysfunction (class III, $n=4$ ). The 2-year OS of each RPA class was $65.5 \%$ in class I, $38.0 \%$ in class II, and $25.0 \%$ in class III and was statistically significant ( $\mathrm{p}=0.001$ ) (Fig. 2D).

\section{Toxicity}

During IMRT-based reirradiation, no severe acute toxicity was recorded. At a median of 18.5 months after retreatment, grade $\geq 3$ toxicity developed in 10 patients $(8.5 \%)$, which consisted of grade 3 mucositis ( $n=2)$, interorgan fistula $(n=4)$, dysphagia $(\mathrm{n}=2)$, and osteoradionecrosis $(\mathrm{n}=1)$ and grade 5 carotid blowout $(\mathrm{n}=1)$. With respect to organ dysfunction, 6 patients $(5.1 \%)$ were tracheostomy-dependent, while 5 patients $(4.2 \%)$ were feeding tube-dependent (Table 3$)$.

\section{Discussion}

Our multi-center cohort study showed a 2-year OS of 43.1\% and a 2-year LC of $53.5 \%$ after IMRT-based reirradiation for RSPHNC. The RSPHNC patients with small size tumors, a resectable status, and long interval between RT courses had a significantly better survival than their counterparts. We also validated the MIRI RPA classification by showing that RPA class I had a higher OS than class II or III. Regarding LC, we identified the independent impact of the interval between RT courses and performance of salvage surgery.

Previously published literature on IMRT for HNC reported that IMRT provides better oncologic outcomes and less toxicities than conventional RT [14,15]. Extending that view- 
A

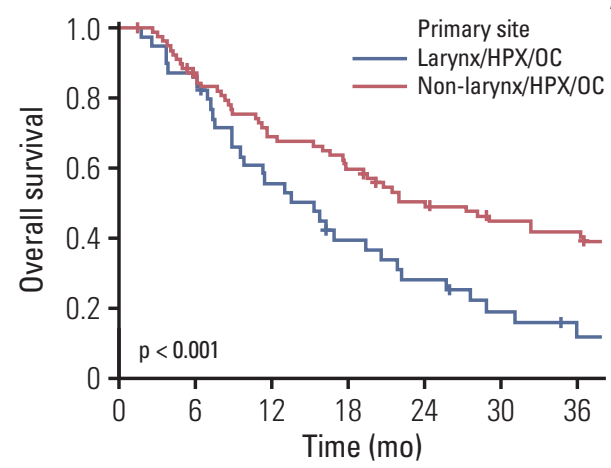

C

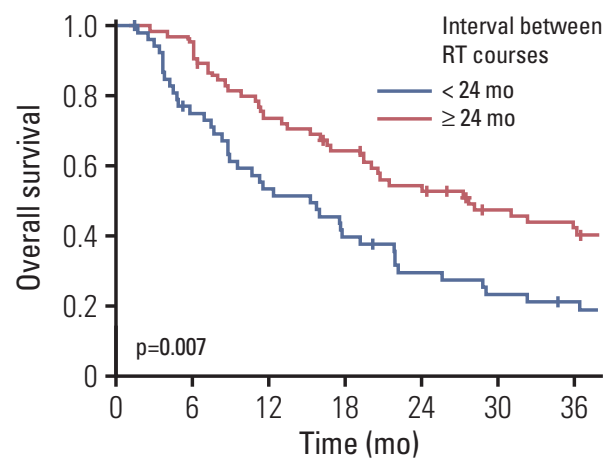

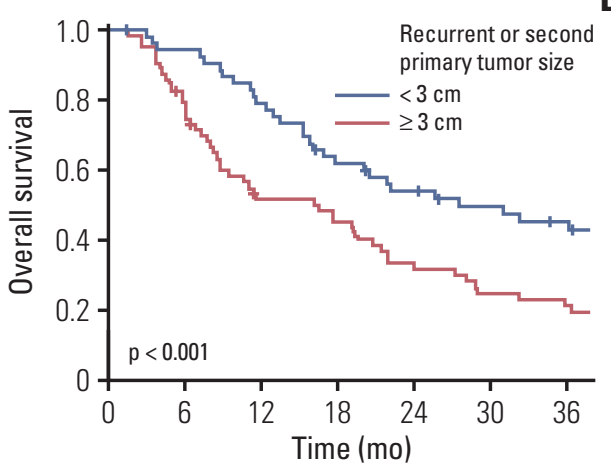

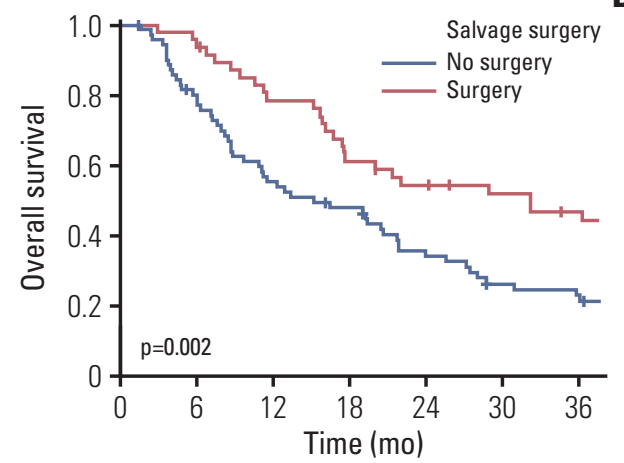

B

D

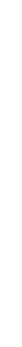

E

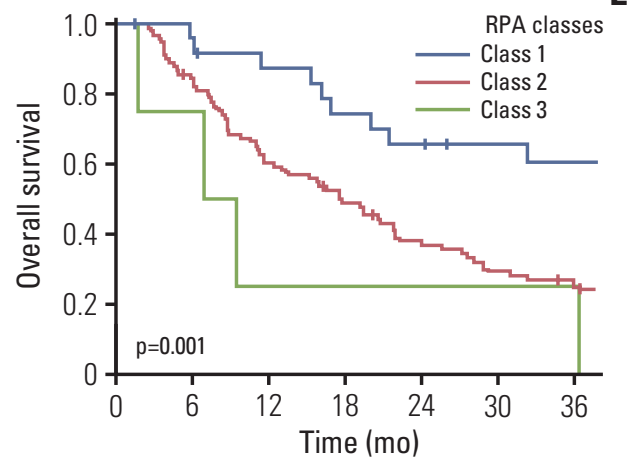

Fig. 2. Kaplan-Meier curve of overall survival rate according to primary subsite (A), tumor size of recurrent or second primary tumor (B), interval between radiotherapy (RT) courses (C), salvage surgery (D), and recursive partitioning analysis (RPA) classes defined by the Multi-Institution Reirradiation Collaborative (E).

point for RSPHNC patients, survival after IMRT-based reirradiation showed an improvement compared to conventional conformal reirradiation. The landmark prospective reirradiation trials in the pre-IMRT era including RTOG 9610 [16] and RTOG 9911 [17] reported that the 2-year OS ranged from 15\% to $25 \%$ in patients who did not undergo salvage surgery. On the contrary, the MIRI group representatively showed an OS rate of $40 \%$ at 2 years following reirradiation of $\mathrm{HNC}$ in the IMRT era, which was $45 \%$ for postoperative patients and $36 \%$ for definitive patients [13]. Additionally, the Italy head and neck working group reported excellent outcomes of $44 \%$ OS at 5-year after reirradiation using advanced RT [18]. Of note, the current study showed that OS at 2 years was $43 \%$, which was consistent with the abovementioned studies involving IMRT-based reirradiation. The favorable OS in our study could have resulted not only from this modern advanced RT technique but also from the high proportion of patients in RPA class I and II (96\%), those who underwent salvage surgery $(41 \%)$, those with a longer interval RT course application (56\%), and those with no organ dysfunction at reirradiation $(93 \%)$.

Specifically, our data supported that salvage surgery in 


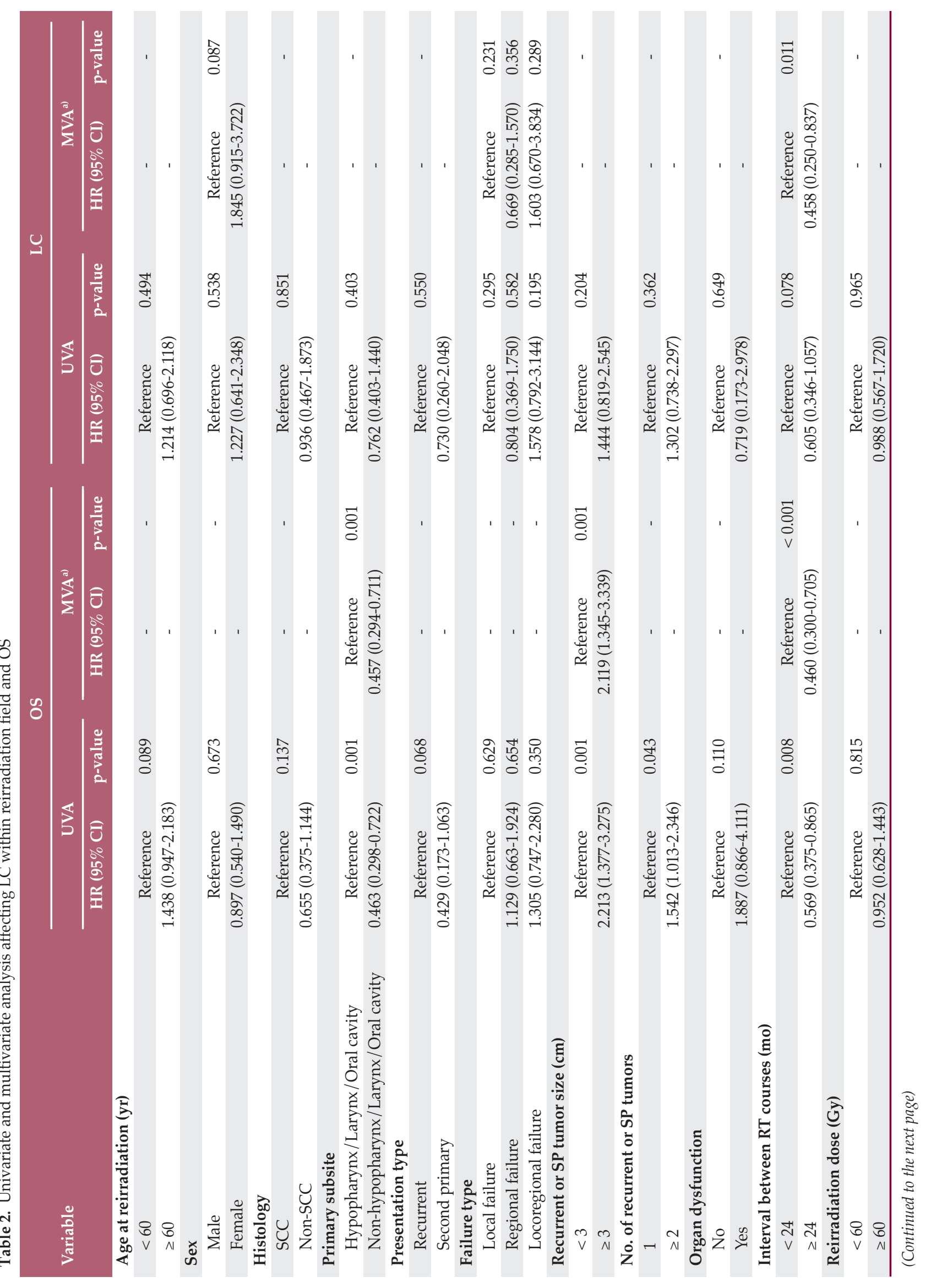




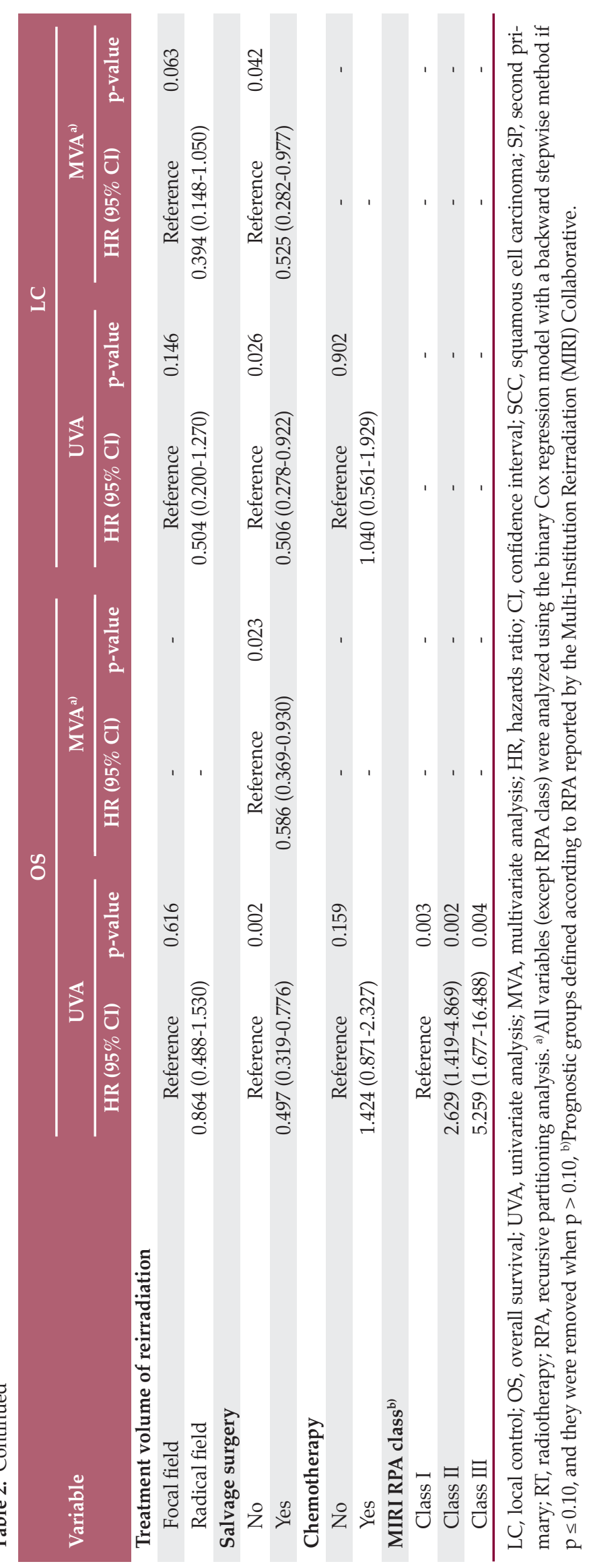


A

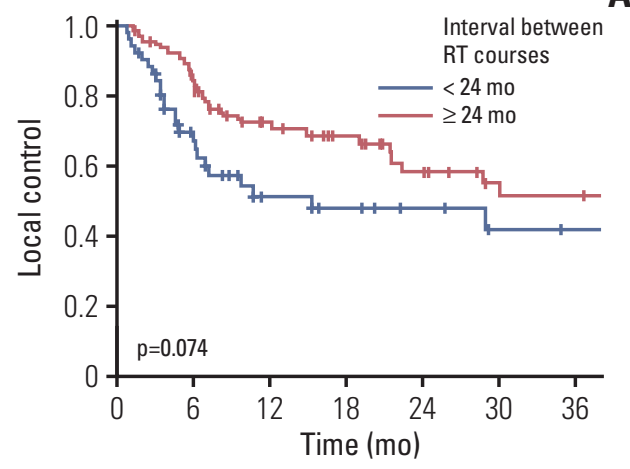

B

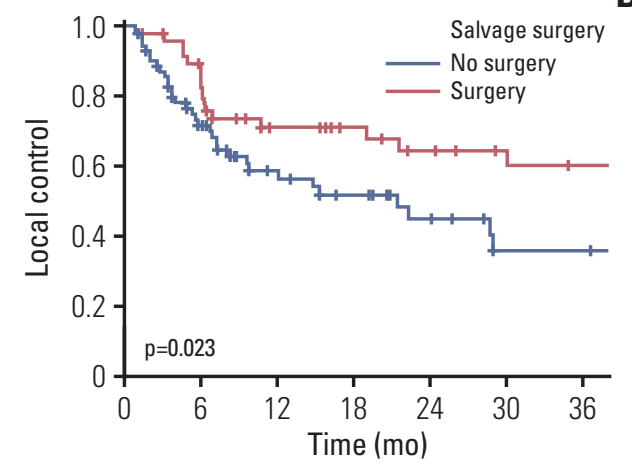

Fig. 3. Kaplan-Meier curve of local control rate according to interval between radiotherapy (RT) courses (A) and salvage surgery (B).

conjunction with IMRT reirradiation for RSPHNC improved OS and LC $[13,19]$. Surgical resection of RSPHNC could be an important factor considering that RSPHNC was generated from radioresistant clonogens after initial chemoradiation [20,21]. Finally, removing macroscopic tumors is a way to enhance retreatment effectiveness owing to the limited dose of IMRT-based reirradiation for gross tumors since the adjacent organ around the tumor was already irradiated with a high dose during previous RT. It was suggested that when patients with RSPHNC expected medically operable and convincing problem, salvage surgery should be encouraged cautiously. The higher LC and OS were the result of the longer interval between RT courses. The appropriate time interval between RT treatments for performing reirradiation is not established, although it depends on the relation between previous the irradiated dose to organs at risk and its tolerance dose associated with normal tissue damage repair $[22,23]$. Previous studies reported that a longer interval from the previous RT course contributed to improved outcomes $[13,21,24,25]$. This was explained by the fact that, the longer the interval between RT treatments, the greater the likelihood of LC and the lesser the aggressiveness of recurrent disease. It is noteworthy that in our patient population, organ dysfunction was not a part of prognostic factors affecting OS since we had only $6.8 \%$ of all patients with organ dysfunction. It shows that there was a bias in patient selection for reirradiation at each institution. It could be presumed that a patient without organ dysfunction was selected for retreatment with IMRT-based reirradiation.

We validated the MIRI RPA classification for the whole patients' population. The RPA classification differentiated the survival between class I and II/II. This difference was statistically significant. MIRI RPA class I cohort $(66 \%)$ had a superior 2-year OS than class II and III (38\% and 25\%, respectively) cohorts. MIRI RPA class I might be the ideal subgroup who should undergo the active salvage treatment including IMRT reirradiation and/or surgical resection [13]. We found that the proposed RPA model might be applicable for our
Table 3. Incidence of severe toxicity and organ dysfunction status following IMRT-based reirradiation

\begin{tabular}{|lr|}
\hline Variable & No. $(\%)$ \\
\hline Grade $\geq 3$ toxicity & $10(8.5)$ \\
Grade 3 & \\
\hline Mucositis & $2(1.7)$ \\
\hline Interorgan fistula & $4(3.4)$ \\
\hline Dysphagia & $2(1.7)$ \\
\hline Osteoradionecrosis & $1(0.8)$ \\
\hline Grade 5 & \\
\hline Carotid blowout & $1(0.8)$ \\
\hline Organ dysfunction & $11(9.3)$ \\
\hline Tracheostomy & $6(5.1)$ \\
\hline Feeding tube dependence & $5(4.2)$ \\
\hline
\end{tabular}

IMRT, intensity-modulated radiotherapy.

RSPHNC patient population and may help in patient selection for retreatment.

Reirradiation for RSPHNC is a challenging issue owing to an increased risk of severe toxicities including fatality [23]. The rate of severe and fatal late toxicity in these cohorts was $8.5 \%$ and $0.8 \%$, respectively, whereas previous prospective studies reported the severe toxicity ranging from $22 \%$ to $34 \%$ and fatality rates of $3.6 \%$ to $7.6 \%$, respectively [16,17]. The amelioration of safety was likely attributed to not only the intensity-modulated technique, but also the conservative patient selection criteria of each institution prior to embarking the reirradiation course. The MIRI Collaborative group reported that in the modern reirradiation era, the risk of progression or death $(64 \%)$ is four times the incidence of severe late toxicity following reirradiation (17\%) [26]. Such risk of late toxicity was more dependent on patient or disease factors than treatment factors. This implied that future research related to patient selection benefiting from reirradiation is needed to elicit the effectiveness of reirradiation using modern technology including IMRT. 
This current study has many limitations. The dominant weakness is in its retrospective nature and a heterogeneous population from multi-centers. Our cohort had various features in terms of patient selection and treatment characteristics according to the physician's discretion. Further, other limitations were the small number of patients from each center, which originated from the restriction of the cohort enrollment period, and the inherent limitations of IMRT. We did not identify the prognostic impact of human papillomavirus (HPV) in patients [27], especially those with oropharyngeal cancer undergoing reirradiation due to the availability of the HPV status of only 10 patients.

In conclusion, our multi-institutional study showed that IMRT-based reirradiation with a median dose of $60 \mathrm{~Gy}$ contributed to increased OS for patients with RSPHNC and had acceptable complications. Given the restricted salvage options, it could be considered an effective treatment for
RSPHNC patients, especially those with small resectable tumors and a long interval between RT courses. Additionally, our cohort confirmed the prognostic validity of the survival rate of the MIRI RPA classification.

\section{Electronic Supplementary Material}

Supplementary materials are available at Cancer Research and Treatment website (https://www.e-crt.org).

\section{Conflicts of Interest}

Conflicts of interest relevant to this article was not reported.

\section{Acknowledgments}

This work was supported by Inha University Hospital Research Grant and the National Research Foundation of Korea (NRF) grant funded by the Korean government (MISP) under NRF-2014R1A5A2009392.

\section{References}

1. Chao KS, Ozyigit G, Tran BN, Cengiz M, Dempsey JF, Low DA. Patterns of failure in patients receiving definitive and postoperative IMRT for head-and-neck cancer. Int J Radiat Oncol Biol Phys. 2003;55:312-21.

2. Ang KK, Zhang Q, Rosenthal DI, Nguyen-Tan PF, Sherman EJ, Weber RS, et al. Randomized phase III trial of concurrent accelerated radiation plus cisplatin with or without cetuximab for stage III to IV head and neck carcinoma: RTOG 0522. J Clin Oncol. 2014;32:2940-50.

3. Beitler JJ, Zhang Q, Fu KK, Trotti A, Spencer SA, Jones CU, et al. Final results of local-regional control and late toxicity of RTOG 9003: a randomized trial of altered fractionation radiation for locally advanced head and neck cancer. Int J Radiat Oncol Biol Phys. 2014;89:13-20.

4. Wong LY, Wei WI, Lam LK, Yuen AP. Salvage of recurrent head and neck squamous cell carcinoma after primary curative surgery. Head Neck. 2003;25:953-9.

5. Goodwin WJ Jr. Salvage surgery for patients with recurrent squamous cell carcinoma of the upper aerodigestive tract: when do the ends justify the means? Laryngoscope. 2000; 110:1-18.

6. Cacicedo J, Navarro A, Alongi F, Gomez de Iturriaga A, Del Hoyo O, Boveda E, et al. The role of re-irradiation of secondary and recurrent head and neck carcinomas: is it a potentially curative treatment? A practical approach. Cancer Treat Rev. 2014;40:178-89.

7. Intensity Modulated Radiation Therapy Collaborative Working Group. Intensity-modulated radiotherapy: current status and issues of interest. Int J Radiat Oncol Biol Phys. 2001;51:880914.

8. Puri DR, Chou W, Lee N. Intensity-modulated radiation therapy in head and neck cancers: dosimetric advantages and update of clinical results. Am J Clin Oncol. 2005;28:415-23.

9. Lee N, Puri DR, Blanco AI, Chao KS. Intensity-modulated radiation therapy in head and neck cancers: an update. Head Neck. 2007;29:387-400.

10. Ho JC, Phan J. Reirradiation of head and neck cancer using modern highly conformal techniques. Head Neck. 2018;40: 2078-93.

11. Chen AM, Phillips TL, Lee NY. Practical considerations in the re-irradiation of recurrent and second primary headand-neck cancer: who, why, how, and how much? Int J Radiat Oncol Biol Phys. 2011;81:1211-9.

12. Kim YS. Reirradiation of head and neck cancer in the era of intensity-modulated radiotherapy: patient selection, practical aspects, and current evidence. Radiat Oncol J. 2017;35:1-15.

13. Ward MC, Riaz N, Caudell JJ, Dunlap NE, Isrow D, Zakem SJ, et al. Refining patient selection for reirradiation of head and neck squamous carcinoma in the IMRT era: a multi-institution cohort study by the MIRI collaborative. Int J Radiat Oncol Biol Phys. 2018;100:586-94.

14. Toledano I, Graff P, Serre A, Boisselier P, Bensadoun RJ, Ortholan $\mathrm{C}$, et al. Intensity-modulated radiotherapy in head and neck cancer: results of the prospective study GORTEC 2004-03. Radiother Oncol. 2012;103:57-62.

15. Zhang B, Mo Z, Du W, Wang Y, Liu L, Wei Y. Intensity-modulated radiation therapy versus $2 \mathrm{D}-\mathrm{RT}$ or $3 \mathrm{D}-\mathrm{CRT}$ for the treatment of nasopharyngeal carcinoma: a systematic review and meta-analysis. Oral Oncol. 2015;51:1041-6.

16. Spencer SA, Harris J, Wheeler RH, Machtay M, Schultz C, Spanos W, et al. Final report of RTOG 9610, a multi-institutional trial of reirradiation and chemotherapy for unresectable recurrent squamous cell carcinoma of the head and neck. Head Neck. 2008;30:281-8.

17. Langer CJ, Harris J, Horwitz EM, Nicolaou N, Kies M, Curran $\mathrm{W}$, et al. Phase II study of low-dose paclitaxel and cisplatin in combination with split-course concomitant twice-daily reirradiation in recurrent squamous cell carcinoma of the head and 
neck: results of Radiation Therapy Oncology Group Protocol 9911. J Clin Oncol. 2007;25:4800-5.

18. Orlandi E, Bonomo P, Ferella L, D’Angelo E, Maddalo M, Alterio $\mathrm{D}$, et al. Long-term outcome of re-irradiation for recurrent or second primary head and neck cancer: a multi-institutional study of AIRO-Head and Neck working group. Head Neck. 2019;41:3684-92.

19. Riaz N, Hong JC, Sherman EJ, Morris L, Fury M, Ganly I, et al. A nomogram to predict loco-regional control after re-irradiation for head and neck cancer. Radiother Oncol. 2014;111:382-7.

20. Weichselbaum RR, Beckett MA, Schwartz JL, Dritschilo A. Radioresistant tumor cells are present in head and neck carcinomas that recur after radiotherapy. Int J Radiat Oncol Biol Phys. 1988;15:575-9.

21. Yard B, Chie EK, Adams DJ, Peacock C, Abazeed ME. Radiotherapy in the era of precision medicine. Semin Radiat Oncol. 2015;25:227-36.

22. Van der Schueren E, Van den Bogaert W, Vanuytsel L, Van Limbergen E. Radiotherapy by multiple fractions per day (MFD) in head and neck cancer: acute reactions of skin and mucosa. Int J Radiat Oncol Biol Phys. 1990;19:301-11.

23. Hoebers F, Heemsbergen W, Moor S, Lopez M, Klop M, Tesselaar M, et al. Reirradiation for head-and-neck cancer: delicate balance between effectiveness and toxicity. Int J Radiat Oncol Biol Phys. 2011;81:e111-8.

24. Velez MA, Veruttipong D, Wang PC, Chin R, Beron P, Abemayor E, et al. Re-irradiation for recurrent and second primary cancers of the head and neck. Oral Oncol. 2017;67:46-51.

25. Chopra S, Gupta T, Agarwal JP, Budrukkar A, Ghosh-Laskar S, Dinshaw K. Re-irradiation in the management of isolated neck recurrences: current status and recommendations. Radiother Oncol. 2006;81:1-8.

26. Ward MC, Lee NY, Caudell JJ, Zajichek A, Awan MJ, Koyfman SA, et al. A competing risk nomogram to predict severe late toxicity after modern re-irradiation for squamous carcinoma of the head and neck. Oral Oncol. 2019;90:80-6.

27. Velez MA, Wang PC, Hsu S, Chin R, Beron P, Abemayor E, et al. Prognostic significance of HPV status in the re-irradiation of recurrent and second primary cancers of the head and neck. Am J Otolaryngol. 2018;39:257-60. 